\title{
Measurement of Human Brown Adipose Tissue Volume and Activity Using Anatomic MR Imaging and Functional MR Imaging
}

\author{
Yin-Ching Iris Chen*1,2, Aaron M. Cypess*2,3, Yih-Chieh Chen ${ }^{2,4}$, Matthew Palmer ${ }^{2,4}$, Gerald Kolodny ${ }^{2,4}$, \\ C. Ronald Kahn ${ }^{2,3}$, and Kenneth K. Kwong ${ }^{1,2}$ \\ ${ }^{I}$ Athinoula A. Martinos Center for Biomedical Imaging, Department of Radiology, Massachusetts General Hospital, Charlestown, \\ Massachusetts; ${ }^{2}$ Harvard Medical School, Boston, Massachusetts; ${ }^{3}$ Section of Integrative Physiology and Metabolism, Research \\ Division, Joslin Diabetes Center, Boston, Massachusetts; and ${ }^{4}$ Division of Nuclear Medicine, Department of Radiology, Beth Israel \\ Deaconess Medical Center, Boston, Massachusetts
}

The aim of this study was to assess the volume and function of human brown adipose tissue (BAT) in vivo using MR imaging. Methods: BAT volumes under thermoneutral conditions in the cervical areas were assessed via water-fat contrast using the Dixon method and via water-saturation efficiency using fast spin-echo and T2-weighted images. The existence of cervical BAT was also assessed by ${ }^{18} \mathrm{~F}-\mathrm{FDG}$ PET/CT scans in the same subjects. BAT functionality was assessed via functional MR imaging (fMRI) blood oxygenation level-dependent (BOLD) signal changes in response to a mild cold challenge. Results: Under thermoneutral conditions, we were able to distinguish BAT from white adipose tissue in the cervical and supraclavicular fat. BAT showed higher water-to-fat contrast and higher water-saturation efficiency in MR imaging scans. The location and volume of BAT assessed by MR imaging were comparable to the measurements by ${ }^{18} \mathrm{~F}-\mathrm{FDG}$ PET/CT scans. During mild cold challenge, BOLD fMRI signal increased in BAT by $10.7 \% \pm 1.8 \%$ $(P<0.01)$. Conclusion: We demonstrated the feasibility of using MR imaging and $\mathrm{AMRI}$ to assess BAT volume and BAT responses to mild cold stimulation in the cervical areas of human subjects.

Key Words: ${ }^{18} \mathrm{~F}-\mathrm{FDG}$; MR imaging; fMRl; cold-activation; brown adipose tissue

J Nucl Med 2013; 54:1584-1587

DOI: 10.2967/jnumed.112.117275

B rown adipose tissue (BAT) is a unique organ whose role is to consume fat calories by generating heat in response to cold exposure (1-4). BAT energy expenditure may also be used to counterbalance the accumulation of stored energy in white adipose tissue (WAT), making induction of BAT thermogenesis a promising target for treating obesity and metabolic disease. Unfortunately, these goals cannot be met until BAT volume and activity in humans can be quantified. This quantification has been a challenge because the

Received Nov. 19, 2012; revision accepted Apr. 8, 2013.

For correspondence contact: Yin-Ching Iris Chen, Massachusetts General Hospital and Harvard Medical School, Rm. 2301, Bldg. 149, 13th St., Charlestown, MA 02129.

E-mail: iris@nmr.mgh.harvard.edu

${ }^{*}$ Contributed equally to this work.

Published online Jul. 18, 2013.

COPYRIGHT (C 2013 by the Society of Nuclear Medicine and Molecular Imaging, Inc. only currently available method for imaging BAT uses a combination of ${ }^{18} \mathrm{~F}$-FDG PET and CT, which gives an adequate estimate of activity but is not sensitive to BAT in the thermoneutral state. Using rodent models, we recently showed that a combination of MR imaging and functional MR imaging (fMRI) can be used to measure BAT volume and activity (5). However, the principal rodent interscapular BAT depot lies in a defined anatomic location and is composed homogeneously of brown adipocytes. By contrast, human BAT is a heterogeneous mixture of white and brown adipocytes in multiple sites, making its imaging particularly challenging. In the current work, we demonstrate how MR imaging and fMRI can be used in humans to quantify BAT volume under thermoneutral conditions and also monitor the dynamic changes in BAT blood flow in response to cold stimulation.

The appeal of MR imaging is that it has the potential to distinguish BAT and WAT based on two independent physiologic factors: BAT has a higher water-to-fat ratio than WAT, and BAT has a high density of mitochondria and blood vessels (6-9). Efforts to evaluate animal BAT volume and distribution with in vivo techniques have used MR spectroscopy $(6,10,11)$, MR imaging, and a combination of the two, such as chemical shift imaging (7). These MR studies were based on the fact that BAT differs from WAT by the fraction of saturated fatty acids, diunsaturated fatty acids $(6)$, and water content $(6,7,11)$. However, a reliable MR protocol to measure BAT volume and distribution in living adult humans is still lacking. Here, we used sequences available in most clinical MR scanners to access BAT location and function. This approach is preferable to ${ }^{18} \mathrm{~F}-\mathrm{FDG} \mathrm{PET} / \mathrm{CT}$, which is limited by several factors, a critical one being the requirement to activate the tissue by exposing the human subject to some kind of challenge such as a cold stress. Thus, PET/CT permits the detection of an unknown and variable fraction of the total BAT volume and reveals only the tissue avidly involved in glucose utilization but none of the other potential substrates, such as fatty acids $(12,13)$. On the other hand, MR imaging is capable of assessing BAT volume using water-fat separation and of monitoring BAT activity through the blood oxygenation level-dependent (BOLD) mechanism continuously throughout the period of cold exposure.

\section{MATERIALS AND METHODS}

Five healthy volunteers were recruited for MR imaging and fMRI studies. Four of the 5 subjects underwent a previous ${ }^{18} \mathrm{~F}-\mathrm{FDG}$ PET/CT 
study demonstrating detectable cold-activated BAT in the cervical, supraclavicular, and upper thoracic depots (Supplemental Table 1; supplemental materials are available at http://jnm.snmjournals.org) (14). All MR imaging studies were performed using a 1.5-T Avanto TIM system (Siemens). The test subjects wore a temperaturecontrolled water circulation cooling vest (Polar Products Inc.). The circulating water was set to room temperature $\left(22^{\circ} \mathrm{C}-24^{\circ} \mathrm{C}\right)$ during BAT anatomic scans and during the baseline period of the fMRI scans. The circulating water was switched to $13^{\circ} \mathrm{C}-16^{\circ} \mathrm{C}$ to provide mild cold stimulation during fMRI scans. In euthyroid volunteers, the temperature used in the vest did not induce shivering, which was confirmed by regular questioning of the subjects during the scan. This study followed institutional guidelines and was approved by the ethics committees of Partners Health Care, Beth Israel Deaconess Medical Center, and Joslin Diabetes Center. Written informed consent was obtained from all volunteers.

\section{MR Imaging Anatomic Scans for Locating BAT}

All anatomic scans were acquired at a spatial resolution of $1.48 \times$ $1.48 \times 2.5 \mathrm{~mm}^{3}$. BAT mass was assessed via 2 methods: water-saturation (WS) efficiency and water-to-fat contrast ratio, because the high water-to-fat ratio in BAT makes a water-suppression-based WS routine more effective in BAT than in WAT (15). Thus, BAT mass was segmented from WAT mass by contrasting signal intensity (SI) with and without water suppression (Eq. 1). WS contrast was obtained from a pair of multislice fast spin-echo (FSE) images (repetition time [TR], 3,000 ms; effective echo time [TE], $98 \mathrm{~ms}$ ) with and without WS and also from a pair of T2-weighted (T2W) images (TR, 1,990 ms; TE, $8.4 \mathrm{~ms}$ ) with and without WS. Only the fat areas with significant WS contrast ( $>10 \%$ WS contrast) from both FSE and $\mathrm{T} 2 \mathrm{~W}$ were regarded as BAT to exclude false signal contrast such as from blood vessels.

$$
\begin{aligned}
\text { WS contrast }= & {[\mathrm{SI}(\mathrm{w} / \mathrm{o} \mathrm{WS})} \\
& -\mathrm{SI}(\mathrm{w} \mathrm{WS})] / \mathrm{SI}(\mathrm{w} / \mathrm{o} \mathrm{WS}) \times 100(\%) . \quad \text { Eq. } 1
\end{aligned}
$$

In addition to WS contrast, BAT was distinguished from WAT using the Dixon method (TR, $6.59 \mathrm{~ms}$; TEs, 2.38 and $4.76 \mathrm{~ms}$ ) (16). The Dixon method separates fat from water by acquiring 2 images in the same scan, one at an echo time when fat and water signals are in phase and another when fat and water signals are out of phase. Water-only and fat-only images are then generated mathematically from these 2 sets of images. Because water content is higher in BAT than in WAT, BAT mass thus has higher water-to-fat contrast (Eq. 2):

$$
\text { water-to-fat contrast }=\mathrm{SI}_{\mathrm{water}} / \mathrm{SI}_{\mathrm{fat}} \text {. }
$$

We tested the water-to-fat contrast method in 3 subjects to identify BAT mass at thermoneutral state.

FSE and T2W were postregistered to the Dixon images using FLIRT, a rigid registration routine in FsFast (FreeSurfer Functional Analysis Stream; MGH). The Dixon fat image was used to exclude nonfat signals for all anatomic scans. Subcutaneous fat was manually segmented and excluded from BAT analysis. Both WS efficiency and water-fat contrast were calculated according to Equations 1 and 2 on a pixel-by-pixel basis.

\section{fMRI Scans for Probing BAT Activity to Cold Challenge}

BAT responding to cold challenge was assessed using a 3dimensional (3D) fast low-angle shot (FLASH) sequence repetitively (TR, $20 \mathrm{~ms}$; TE, $1.85 \mathrm{~ms}$ ). Two baseline points were acquired before the switch to cold challenge $\left(13^{\circ} \mathrm{C}-16^{\circ} \mathrm{C}\right)$ and were followed by repetitive 3D FLASH scans over the next $30 \mathrm{~min}$. fMRI data were motion-corrected and registered to Dixon images, masked by Dixon fat images to exclude signal changes from nonfat areas. The map of percentage signal changes to cold challenge was masked by statistical significance of a Student's $t$ test $(P<0.01)$.

\section{RESULTS}

The WS efficiency approach used two independent MR imaging sequences: a conventional T2W spin-echo sequence and an FSE sequence. A color map shows the fat area where the watersuppression analysis led to significant signal reduction (WS contrast $>10 \%$ ) and thus the location of BAT (Fig. 1A). Similarly, the water-to-fat contrast method via the Dixon method also detected BAT mass in the same areas (Fig. 1B). The BAT mass located by MR imaging was matched with previous cold-activated

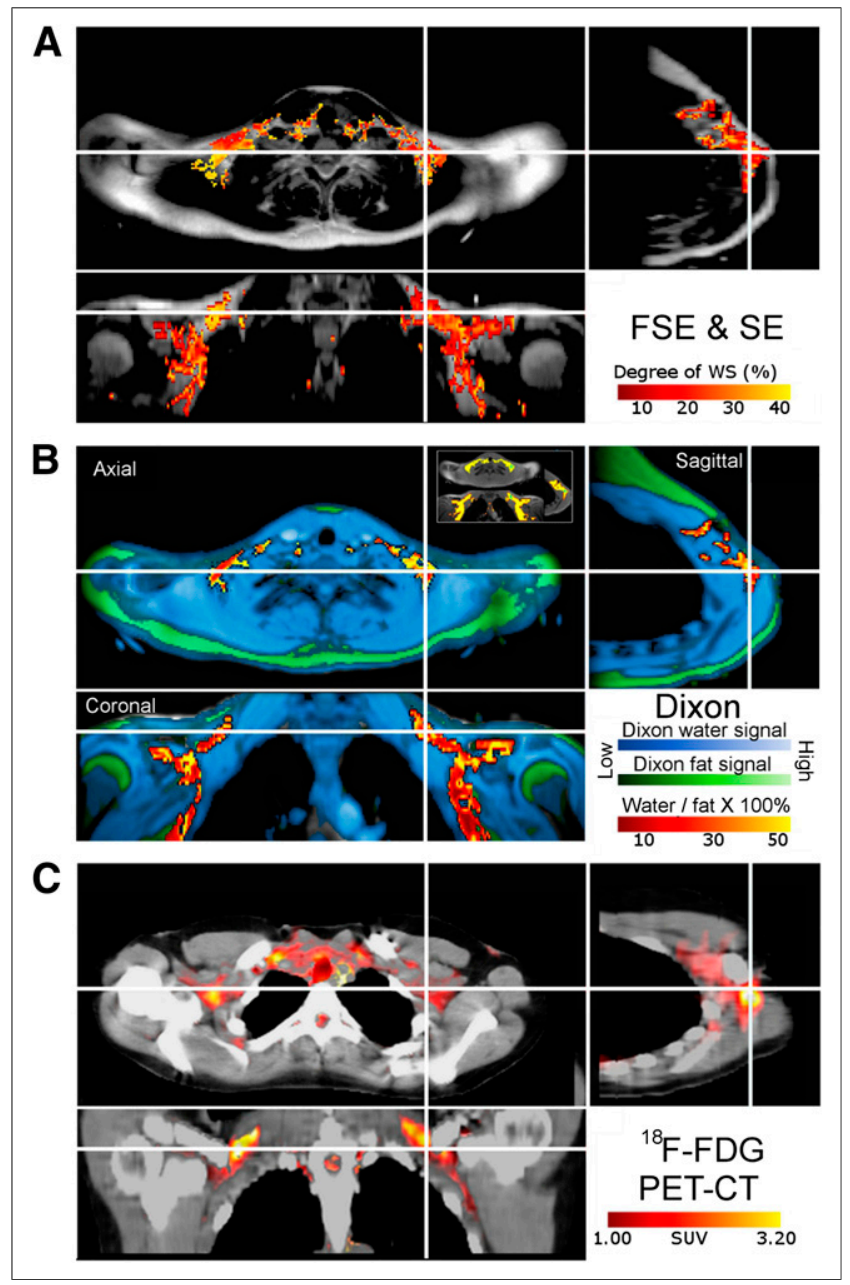

FIGURE 1. (A) Detecting BAT mass (color overlay) by watersuppression efficiency using traditional spin-echo (SE) images and FSE images. Color map was WS contrast, masked by mask generated from overlapping masks of FSE and SE methods. (B) Detecting BAT mass (red-yellow overlay) via water-fat contrast using Dixon method. Blue tone $=$ water image; green tone $=$ fat image; red = water-to-fat contrast (indicator for BAT). Insert showed nonsubcutaneous fat mask generated by Dixon method. (C) BAT was located via cold-activated ${ }^{18} \mathrm{~F}-\mathrm{FDG}$ PET imaging. MR imaging and PET methods detected BAT mass at similar locations (e.g., cross hairs). All images were from same subject but with slight anatomic differences between PET and MR imaging due to arm-up (PET) versus arm-down (MR imaging) posture. Images were from subject 1 (Table 1). 
TABLE 1

Estimated BAT Volume $(\mathrm{mL})$

\begin{tabular}{|c|c|c|c|}
\hline \multirow[b]{2}{*}{ Subject no. } & \multicolumn{2}{|c|}{ MR imaging } & \multirow[b]{2}{*}{${ }^{18} \mathrm{~F}-\mathrm{FDG} \mathrm{PET} / \mathrm{CT}$ imaging } \\
\hline & WS contrast, FSE and SE & Water-fat ratio, Dixon & \\
\hline \multicolumn{4}{|l|}{1} \\
\hline Left & 18.1 & 19.5 & 20.2 \\
\hline Right & 21.4 & 24.8 & 21.4 \\
\hline \multicolumn{4}{|l|}{2} \\
\hline Left & 22.6 & 18.2 & 22.4 \\
\hline Right & 21.0 & 14.7 & 20.1 \\
\hline \multicolumn{4}{|l|}{3} \\
\hline Left & 13.8 & Not applicable & 27.8 \\
\hline Right & 14.8 & Not applicable & 28.9 \\
\hline \multicolumn{4}{|c|}{ 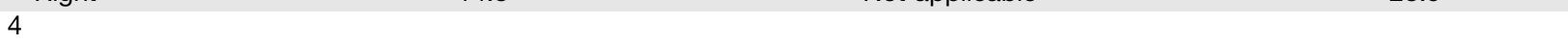 } \\
\hline Left & 23.3 & Not applicable & 28.9 \\
\hline Right & 29.6 & Not applicable & 28.6 \\
\hline \multicolumn{4}{|l|}{5} \\
\hline Left & 21.5 & 24.3 & Not applicable \\
\hline Right & 27.4 & 31.0 & Not applicable \\
\hline
\end{tabular}

Only areas meeting WS contrast criteria (>10\%) for FSE and SE method were counted toward BAT volume.

${ }^{18} \mathrm{~F}-\mathrm{FDG}$ PET/CT results in 4 of the subjects $(1$ subject did not undergo a PET/CT study [Fig. 1C]). Both MR imaging and coldactivated ${ }^{18} \mathrm{~F}$-FDG PET/CT studies yielded similar volumes of BAT in the cervical, supraclavicular, and upper thoracic depots (repeated measures ANOVA $P=0.11$ ) (volume estimation, Table 1). The degree to which the different methods demonstrate the cervical and supraclavicular BAT distribution can be appreciated through a 3D reconstruction (Fig. 2; Supplemental Videos 1-3). 3D PET/CT images were generated using PET/CT Viewer freeware (17). MR imaging methods also identified BAT in the deeper cervical depots, such as the carotid sheath, that have been seen using anatomic dissection (18) but were uncertain in the coldactivated PET/CT study.

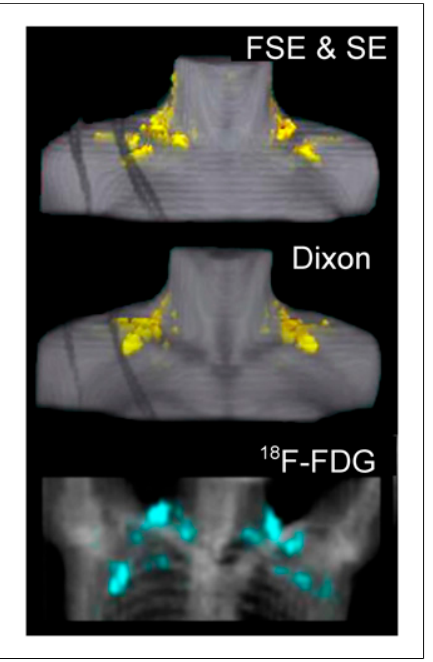

FIGURE 2. 3D reconstruction of BAT mass using MR imaging (with FSE and SE and Dixon) and PET/ CT (subject 2, Table 1). a water-circulating vest $\left(13^{\circ} \mathrm{C}-16^{\circ} \mathrm{C}\right)(14)$ for 60 min while the patient was in the MR imaging scanner $(n=3$ studied). The BOLD signal increased in BAT by $10.7 \% \pm 1.8 \%(P<0.01)$
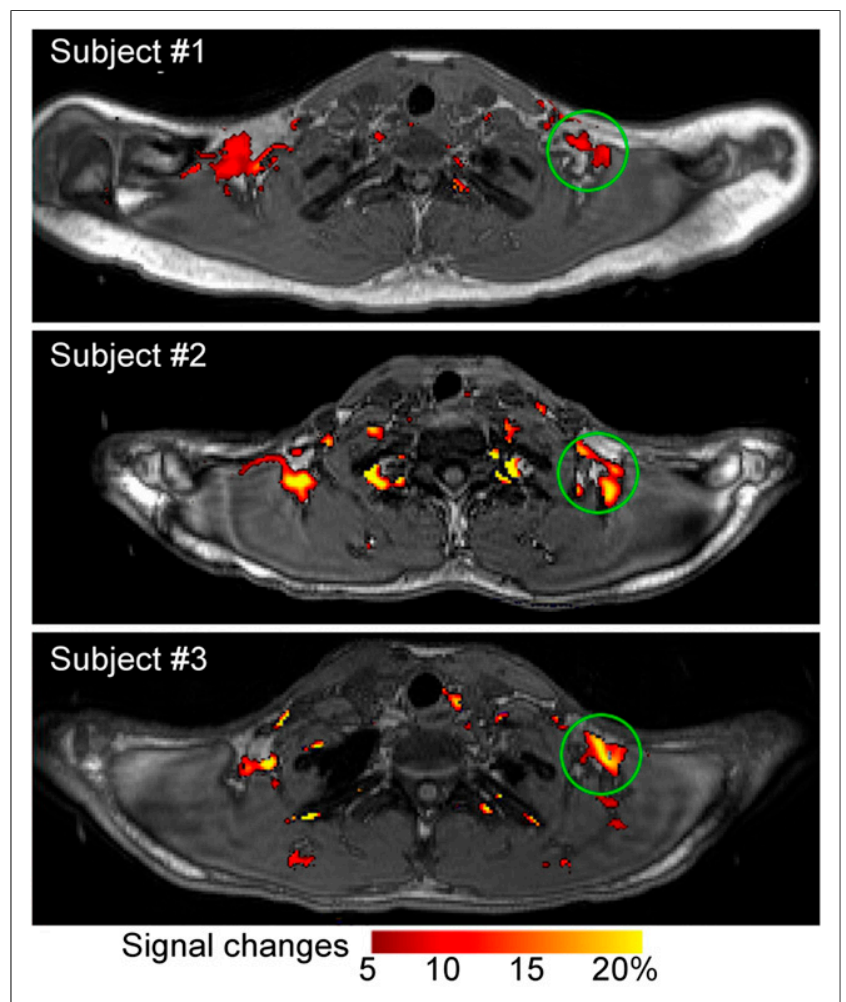

FIGURE 3. fMRI detection of BAT activity on cold challenge $\left(13^{\circ} \mathrm{C}-\right.$ $16^{\circ} \mathrm{C}$ ). Degree of BOLD signal changes (red-yellow map) is superimposed on anatomic images (in gray scale). On cold stimulation, significant BOLD signal increases were found in regions identified as having BAT for subjects 1-3 (such as areas indicated by green circles). Subject 1 is same subject as in Figure 1. 
on cold exposure. The degree of BOLD signal changes was much larger than in the typical brain fMRI study. The high BOLD signal changes in BAT were likely due to larger hemodynamic response to fulfill a higher metabolic demand in BAT and also decreased T1 relaxation time resulting from increased tissue temperature due to BAT thermogenesis.

\section{DISCUSSION}

Understanding human BAT physiology requires an accurate measurement of its volume in addition to its activity in response to stimulation, and we show here how MR imaging and fMRI provide several potential advantages over the current standard, ${ }^{18} \mathrm{~F}-\mathrm{FDG}$ PET/CT. The heterogeneous composition of BAT means that in the thermoneutral resting state PET/CT does not have the resolution to distinguish BAT from WAT. In contrast, the MR sequences used here rely on water content, which can detect BAT even without stimulation.

In our BAT volume estimation, the Dixon fat image was used as a mask to exclude nonfat tissue. Because the estimation of BAT volume was further estimated via WS and fat-water contrast, the fat mask from the Dixon method serves as a rough guideline and has minimal impact on the final BAT volume estimation. Even in the case that the fat tissue was underestimated by the Dixon mask, the primary BAT region in our study appeared to be consistent with the PET/CT measurement.

Cold-activated glucose uptake, as measured by accumulation of ${ }^{18} \mathrm{~F}-\mathrm{FDG}$, does distinguish BAT from WAT, and because it correlates with blood flow (20), it is a useful measure of BAT activity. However, the ultimate fate of glucose in BAT and how well it predicts the amount of thermogenesis or BAT volume has not been established and may have substantial interindividual variation. One cannot automatically assume a proportionate correlation between ${ }^{18}$ F-FDG PET/CT activity and BAT volume measured by MR imaging, even though the two methods localized BAT to similar anatomic sites in our healthy volunteers. Future research should evaluate with greater precision how glucose uptake correlates with BAT water content and hemodynamic parameters evaluated by BOLD, which could be determined through the application of PET/MR imaging. With the ability to measure human BAT volume and activity using nonionizing fMRI, it will now be possible to study BAT physiology serially and in much more diverse populations.

\section{CONCLUSION}

We demonstrate the feasibility of using MR imaging and fMRI to assess BAT volume and BAT responses to mild cold stimulation in the cervical and supraclavicular regions of human subjects.

\section{DISCLOSURE}

The costs of publication of this article were defrayed in part by the payment of page charges. Therefore, and solely to indicate this fact, this article is hereby marked "advertisement" in accordance with 18 USC section 1734. This work was supported by NIH grants DK087317, DK055545, DK033201, DK081604, DK046200, RR025757, and P30 DK036836; the Clinical Translational Science Award UL1RR025758 to Harvard University and BIDMC from the National Center for Research Resources (NCRR), Harvard Catalyst; the Harvard Clinical and Translational Science Center (NIH award no. UL1 RR 025758 and financial contributions from
Harvard University and its affiliated academic health care centers); and the Eli Lilly Foundation. The content is solely the responsibility of the authors and does not necessarily represent the official views of Harvard Catalyst, Harvard University, and its affiliated academic health care centers, NCRR, or NIH. No other potential conflict of interest relevant to this article was reported.

\section{ACKNOWLEDGMENTS}

We thank the support provided by the BIDMC Clinical Research Center nursing team, Bionutrition Core, research pharmacy, and nuclear medicine technologists; Ilan Tal for the development of the PET/CT Viewer software and code for the 3D Viewer; Cathy Sze and Ke Wang for their assistance in recruiting volunteers; and our volunteers for their commitment to the studies.

\section{REFERENCES}

1. Nedergaard J, Golozoubova V, Matthias A, Asadi A, Jacobsson A, Cannon B. UCP1: the only protein able to mediate adaptive non-shivering thermogenesis and metabolic inefficiency. Biochim Biophys Acta. 2001;1504:82-106.

2. Shabalina IG, Kramarova TV, Nedergaard J, Cannon B. Carboxyatractyloside effects on brown-fat mitochondria imply that the adenine nucleotide translocator isoforms ANT1 and ANT2 may be responsible for basal and fatty-acid-induced uncoupling respectively. Biochem J. 2006;399:405-414.

3. Matthias A, Ohlson KB, Fredriksson JM, Jacobsson A, Nedergaard J, Cannon B. Thermogenic responses in brown fat cells are fully UCP1-dependent: UCP2 or UCP3 do not substitute for UCP1 in adrenergically or fatty acid-induced thermogenesis. J Biol Chem. 2000;275:25073-25081.

4. Rial E, Gonzalez-Barroso MM, Fleury C, Bouillaud F. The structure and function of the brown fat uncoupling protein UCP1: current status. Biofactors. 1998;8:209-219.

5. Chen YI, Cypess AM, Sass CA, et al. Anatomical and functional assessment of brown adipose tissue by magnetic resonance imaging. Obesity (Silver Spring). 2012;20:1519-1526.

6. Strobel K, van den Hoff J, Pietzsch J. Localized proton magnetic resonance spectroscopy of lipids in adipose tissue at high spatial resolution in mice in vivo. J Lipid Res. 2008;49:473-480.

7. Hu HH, Smith DL Jr, Nayak KS, Goran MI, Nagy TR. Identification of brown adipose tissue in mice with fat-water IDEAL-MR imaging. J Magn Reson Imaging. 2010;31:1195-1202.

8. Henkelman RM, Hardy PA, Bishop JE, Poon CS, Plewes DB. Why fat is bright in RARE and fast spin-echo imaging. J Magn Reson Imaging. 1992;2:533-540.

9. Zingaretti MC, Crosta F, Vitali A, et al. The presence of UCP1 demonstrates that metabolically active adipose tissue in the neck of adult humans truly represents brown adipose tissue. FASEB J. 2009;23:3113-3120.

10. Branca RT, Warren WS. In vivo brown adipose tissue detection and characterization using water-lipid intermolecular zero-quantum coherences. Magn Reson Med. 2011;65:313-319.

11. Hamilton G, Smith DL Jr, Bydder M, Nayak KS, Hu HH. MR properties of brown and white adipose tissues. J Magn Reson Imaging. 2011;34:468-473.

12. Bartelt A, Bruns OT, Reimer R, et al. Brown adipose tissue activity controls triglyceride clearance. Nat Med. 2011;17:200-205.

13. Ouellet V, Labbe SM, Blondin DP, et al. Brown adipose tissue oxidative metabolism contributes to energy expenditure during acute cold exposure in humans. J Clin Invest. 2012;122:545-552.

14. Cypess AM, Chen YC, Sze C, et al. Cold but not sympathomimetics activates human brown adipose tissue in vivo. Proc Natl Acad Sci USA. 2012;109:10001-10005.

15. da Motta AC, Tunkel DE, Westra WH, Yousem DM. Imaging findings of a hibernoma of the neck. AJNR. 2006;27:1658-1659.

16. Dixon WT. Simple proton spectroscopic imaging. Radiology. 1984;153:189-194.

17. Barbaras L, Tal I, Palmer MR, Parker JA, Kolodny GM. Shareware program for nuclear medicine and PET/CT PACS display and processing. AJR. 2007;188:W565-568.

18. Heaton JM. The distribution of brown adipose tissue in the human. J Anat. 1972;112:35-39.

19. Harris WH, Foster DO, Ma SW, Yamashiro S, Langlais-Burgess LA. The noradrenaline content and innervation of brown adipose tissue in the young rabbit. Can J Physiol Pharmacol. 1986;64:561-567.

20. Orava J, Nuutila P, Lidell ME, et al. Different metabolic responses of human brown adipose tissue to activation by cold and insulin. Cell Metab. 2011;14:272-279. 